\title{
Stroke Patients with Atrial Fibrillation Treated with Oral Anticoagulants: Comparison of the Population-Based Stroke Registry of Dijon and the French National Health Databases
}

\author{
Amélie Gabet $^{\mathrm{a}} \quad$ Valérie Oliéa $^{\mathrm{a}}$ Yannick Béjot ${ }^{\mathrm{b}}$ \\ aNon-Communicable Diseases Department, Santé Publique France, Saint-Maurice, France; \\ ${ }^{b}$ Neurology Unit, Dijon University Hospital, Dijon Stroke Registry, Dijon, France
}

\section{Keywords}

Stroke · Atrial fibrillation · Epidemiology · Population-based registry $\cdot$ Administrative database

\begin{abstract}
Introduction: The objective of this study was to evaluate the complementarity of the French national health database (Système national des données de Santé, SNDS) and the Dijon Stroke Registry for the epidemiology of stroke patients with anticoagulated atrial fibrillation (AF). Methods: The SNDS collects healthcare prescriptions and procedures reimbursed by the French national health insurance for almost all of the 66 million individuals living in France. A previously published algorithm was used to identify AF newly treated with oral anticoagulants. The Dijon Stroke Registry is a population-based study covering the residents of the city of Dijon since 1985 and records all stroke cases of the area. We compared the proportions of stroke patients with anticoagulated AF in the city of Dijon identified in SNDS databases to those registered in the Dijon Stroke Registry. Results: For the period 2013-2017 in the city of Dijon, 1,146 strokes were identified in the SNDS and 1,188 in the registry. The proportion of strokes with anticoagulated AF was $13.4 \%$ in the
\end{abstract}

karger@karger.com

(c) 2020 S. Karger AG, Basel

www.karger.com/ned

Karger"
SNDS and $20.3 \%$ in the Dijon Stroke Registry. Very similar characteristics were found between patients identified through the 2 databases. The overall prevalence of $A F$ in stroke patients could be estimated only in the Dijon stroke registry and was $30.4 \%$ for the study period. Discussion/ Conclusion: If administrative health databases can be a useful tool to study the epidemiology of anticoagulated AF in stroke patients, population-based stroke registries as the Dijon Stroke Registry remain essential to fully study the epidemiology of strokes with anticoagulated AF.

(c) 2020 S. Karger AG, Basel

\section{Introduction}

Atrial fibrillation (AF) is now the leading cause of ischemic stroke in Western countries [1]. However, evaluating the epidemiology of AF in stroke patients is challenging due to the limited exhaustive source data at a national level. In France, the French national health database (Système national des données de Santé, SNDS) collects medical information on the general population residing in France [2]. This massive database exhaustively compiles patients' medical data, including hospital discharge

Amélie Gabet

Santé Publique France Non-Communicable Diseases Department

14 rue du Val d'Osne

FR-94410 Saint Maurice (France)

amelie.gabet@santepubliquefrance.fr 


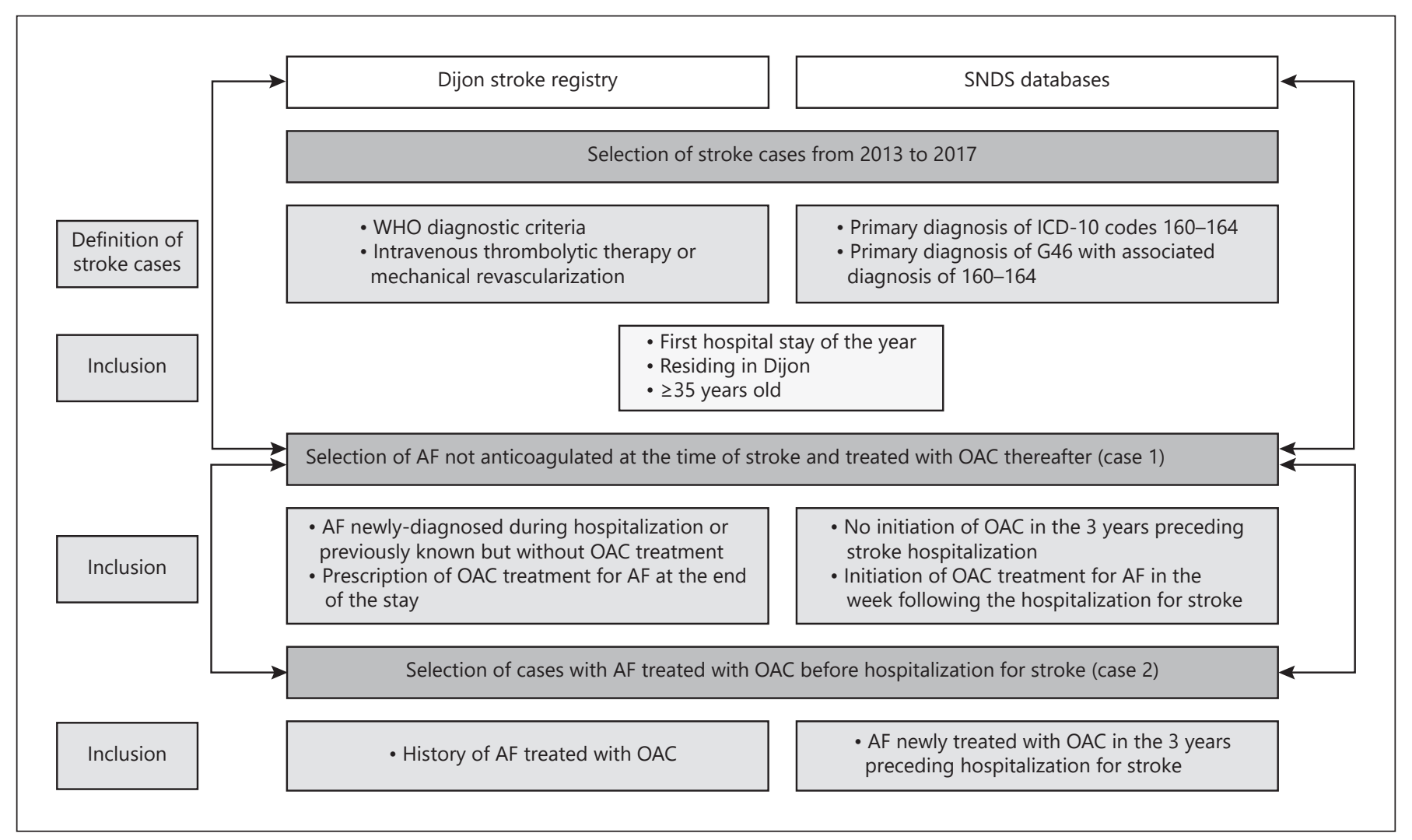

Fig. 1. Flow chart of stroke population selection in the Dijon Stroke registry and in the SNDS for the city of Dijon.

and drug delivery information. Although stroke patients as well as patients treated with oral anticoagulants (OACs) for AF appear to be quite reliably identified in this database, the performance of this tool remains to be investigated. The population-based stroke registry of Dijon, France, exhaustively records all cases of stroke with associated medical information and includes a proper validation. Although the registry covers a small geographical area, it represents the gold standard for stroke epidemiology in France and could therefore be useful to estimate AF-related strokes.

The objective of this study was to evaluate the complementarity of the SNDS databases and the Dijon Stroke Registry for AF surveillance among stroke patients. We compared stroke patients with OAC-treated AF in the city of Dijon identified in SNDS databases to those registered in the Dijon Stroke Registry and report the overall proportion of $\mathrm{AF}$ in stroke patients from the registry. The proportion of stroke patients with OAC-treated AF prior to their stroke and the proportion with AF discovered at the time of stroke and subsequently treated were also compared between the 2 sources.

Stroke with Anticoagulated Atrial Fibrillation

\section{Methods}

\section{Population Selection}

The flow chart (Fig. 1) summarizes the selection of populations. Stroke patients aged over 35 years were identified in the SNDS and the Dijon Stroke Registry. The patient population was limited to hospitalized patients given that out-of-hospital strokes could not be identified in the SNDS but only in the Dijon Stroke Registry in which they account for approximately $4 \%$ of all strokes. Only the first stroke of each calendar year was retained in each database.

\section{Identification of Stroke Cases with AF Treated with OAC} SNDS

The SNDS collects healthcare prescriptions and procedures reimbursed by the French national health insurance for almost all of the 66 million individuals living in France although it does not provide any clinical data concerning examination results, medical visits, and prescriptions (see online suppl. data; for all online suppl. material, see www.karger.com/doi/10.1159/000511206). The national hospital discharge database (Program de médicalisation des systèmes d'information, PMSI), which is part of the SNDS, contains the medical diagnoses for all public and private hospital stays in France. Here, the study population was limited to people living in the city of Dijon.

First, patients hospitalized for stroke were selected for each year from 2013 to 2017 in the SNDS. Stroke was defined by ICD-10 
codes I60 to I64 as the primary diagnosis or G46 as the primary diagnosis with I60-64 as the secondary diagnosis [3, 4]. The primary diagnosis considered was for either the entire hospital stay or one of the medical units where the patient stayed.

Second, patients newly treated with OACs for AF were directly identified in the SNDS if they were newly treated with OACs in the 3 years preceding the stroke hospitalization and had a history of hospitalization for AF, long-term AF disease status, or AF-related medical procedure (ablation or cardioversion) at the time of OAC initiation. OAC treatments delivered to the patients were identified exhaustively as they are systematically reimbursed by the French social security system. If the patients were treated with OACs but the hospital stay did not mention an AF diagnosis, ablation, or long-term AF disease status, we indirectly identified patients treated with OACs for AF using a multiple imputation process detailed in a previous study [5]. This was based on a logistic regression constructed with the following AF predictors: age; sex; delivery of antiarrhythmic drugs, antihypertensive drugs, or $\beta$-blockers; Holter examination; echocardiography; thyroid dosages; and previous hospitalization for arterial diseases [5].

We considered that AF not treated with OACs at the time of stroke - including both AF newly diagnosed at time of stroke and AF previously known but not anticoagulated (case 1) if no OAC was reported for an AF indication prior to the stroke, and an $\mathrm{OAC}$ was initiated for AF in the week following the end of the hospitalization for stroke. Sensitivity analyses were conducted by enlarging this window from 1 week to 1 month. We considered a stroke patient to be previously treated for AF (case 2) if he/she initiated $\mathrm{OAC}$ treatment for $\mathrm{AF}$ in the 3 years prior to the hospitalization for stroke.

\section{Dijon Stroke Registry}

The Dijon Stroke Registry is a population-based study covering the residents of the city of Dijon since 1985 . For this area, the registry exhaustively records all stroke cases (fatal and nonfatal) using multiple sources of information. It complies with the defined criteria for conducting "ideal" incidence stroke studies [6] as well as the guidelines for reporting incidence and prevalence in neuroepidemiology according to Standards of Reporting of Neurological Disorders (STROND).

For this study, only hospitalized stroke patients were selected in the registry for each year from 2013 to 2017. Stroke was defined according to the WHO diagnostic criteria [7]. Patients who received intravenous thrombolytic therapy or mechanical revascularization were considered to have had a stroke even though total recovery was observed within $24 \mathrm{~h}$. A detailed history was obtained from each patient using a standardized questionnaire, and a neurological examination was systematically performed. Data were recorded in the patients' medical files and then reviewed by stroketrained investigators.

AF was either known at the time of stroke or discovered during hospitalization through echocardiography, Holter monitor, or other examinations. Information on patients' prestroke treatment as well as any prescriptions delivered at the end of the hospital stay was recorded. In the Dijon Stroke Registry, data on OAC prescriptions at the end of the hospital stay were available but not on dispensed OAC treatments (i.e., whether the patient actually picked up the treatment in a pharmacy), unlike the SNDS that includes this information. We identified stroke cases with AF not treated with $\mathrm{OAC}$ before hospitalization and prescribed an OAC at the
Table 1. Characteristics of the stroke population residing in Dijon in the French national health database (SNDS) and the Dijon Stroke Registry

\begin{tabular}{lccl}
\hline Source & \multicolumn{3}{l}{ Stroke population } \\
\cline { 2 - 4 } & $\begin{array}{l}\text { SNDS } \\
\text { national }\end{array}$ & $\begin{array}{l}\text { SNDS } \\
\text { Dijon }\end{array}$ & $\begin{array}{l}\text { Dijon Stroke } \\
\text { Registry }\end{array}$ \\
\hline N 2013-2017 & 581,558 & 1,146 & 1,188 \\
Age, mean (SD), years & $75.4(14)$ & $76.2(14)$ & $76.3(14)$ \\
Women, \% & 49.1 & 53.5 & 54.1 \\
Ischemic, \% & 72.2 & 68.6 & 81.1 \\
Hemorrhagic, \% & 24.2 & 26.5 & 17.8 \\
Undetermined, \% & 3.6 & 4.9 & 1.1 \\
\hline
\end{tabular}

SNDS, Système national des données de Santé; SD, standard deviation.

end of the hospital stay (case 1) and stroke cases treated with OAC for AF before stroke (case 2). We also recorded all stroke cases with a history of AF or with AF discovered at the time of hospitalization for stroke, independently of OAC treatments. These patients cannot be identified in the SNDS.

Finally, in both the SNDS and the Dijon Stroke Registry, information is available about stroke patients treated with OAC for AF, with a distinction being made between those with AF not treated with OAC at the time of stroke and treated with OAC after hospitalization for stroke (case 1) and those with a history of AF who were treated with OAC before hospitalization for stroke (case 2). The distribution of age, sex, and stroke subtype was reported. Stroke cases were classified as ischemic, hemorrhagic, or undetermined.

\section{Results}

\section{Stroke Population}

Characteristics of stroke patients are described for the SNDS and Dijon Stroke Registry in Table 1. For the period 2013-2017 in the city of Dijon, 1,146 strokes were identified in the SNDS using the current algorithm and 1,188 in the registry. A similar mean age was recorded in the SNDS (76.2 years) and the registry ( 76.3 years) as well as a similar proportion of women $(53.5$ and $54.1 \%$, respectively). When comparing stroke subtypes, a similar proportion of intracerebral and subarachnoid hemorrhages was found. In the registry, no strokes were classified as undetermined.

\section{Stroke Cases with OAC-Treated AF}

Comparison of the Proportion of Case 1 Patients

For the years 2013-2017, 43 case 1 patients were identified in the SNDS (i.e., 3.8\% of the SNDS stroke popula- 
Table 2. Characteristics of the stroke population with AF treated with OACs in Dijon in the French national health database (SNDS) and the Dijon Stroke Registry

\begin{tabular}{|c|c|c|c|c|c|c|c|c|c|}
\hline \multirow[t]{2}{*}{ Source } & \multicolumn{2}{|c|}{$\begin{array}{l}\text { Stroke patients with AF not treated } \\
\text { with OAC at at the time of stroke and } \\
\text { treated with OAC thereafter (case 1) }\end{array}$} & \multirow{2}{*}{$\begin{array}{l}\text { Dijon stroke registry: } \\
\text { Overall stroke } \\
\text { patients with AF } \\
\text { discovered at the } \\
\text { time of hospitaliza- } \\
\text { tion for stroke }\end{array}$} & \multicolumn{2}{|c|}{$\begin{array}{l}\text { Stroke patients with AF treated } \\
\text { with OAC before hospitalization } \\
\text { for stroke (case 2) }\end{array}$} & \multirow{2}{*}{$\begin{array}{l}\text { Dijon stroke registry: } \\
\text { Overall stroke } \\
\text { patients with a } \\
\text { history of } \mathrm{AF}^{\mathrm{b}}\end{array}$} & \multicolumn{2}{|c|}{$\begin{array}{l}\text { Overall stroke patients with } \\
\text { AF treated with OAC (cases } 1 \\
\text { and } 2 \text { together) }\end{array}$} & \multirow{2}{*}{$\begin{array}{l}\text { Dijon stroke } \\
\text { registry: } \\
\text { Overall stroke } \\
\text { patients with } \mathrm{AF}^{\mathrm{b}}\end{array}$} \\
\hline & SNDS Dijon & $\begin{array}{l}\text { Dijon Stroke } \\
\text { Registry }\end{array}$ & & SNDS Dijon & $\begin{array}{l}\text { Dijon Stroke } \\
\text { Registry }\end{array}$ & & SNDS Dijon & $\begin{array}{l}\text { Stroke Dijon } \\
\text { Registry }\end{array}$ & \\
\hline N 2013-2017 & 43 & 81 & 102 & 111 & 161 & 259 & 154 & 245 & 361 \\
\hline \% Cas 2013-2017 & 3.8 & 6.8 & 8.6 & 9.7 & 13.5 & 21.8 & 13.4 & 20.3 & 30.4 \\
\hline Age, median, years & $76.0(13)$ & $79.3(12)$ & $82.6(12)$ & $80.9(10)$ & $82.4(10)$ & $83.0(10)$ & $79.5(11)$ & $81.4(11)$ & $82.9(11)$ \\
\hline Hemorrhagic, \% & 0.0 & 0 & 5.8 & 24.4 & 23.6 & 18.9 & 17.5 & 15.9 & 15.2 \\
\hline Undetermined, \% & 4.6 & 0 & 2.0 & 9.6 & 1.9 & 1.9 & 8.2 & 1.2 & 1.9 \\
\hline
\end{tabular}

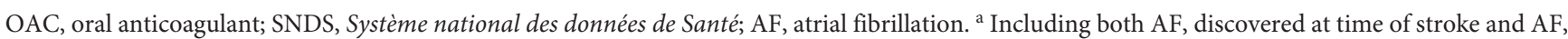
known at time of stroke but not previously anticoagulated. ${ }^{\mathrm{b}}$ Independently of OAC treatment.

tion) and 81 in the Dijon Stroke Registry (i.e., $6.8 \%$ of the registry stroke patients) ( $p=0.002$, Table 2$)$. Age did not differ significantly between the 2 databases, with a mean age of 76.0 and 79.3 years for case 1 patients identified in the SNDS and the registry, respectively $(p=0.29)$.

In the registry, we examined all stroke patients with $\mathrm{AF}$ discovered at the time of stroke and not diagnosed beforehand. These patients accounted for $8.6 \%$ of all strokes, with half of them being prescribed OAC treatment (data not shown).

Comparison of Proportion of Case 2 Patients

We recorded 111 case 2 patients in the SNDS and 161 in the Dijon Stroke Registry, corresponding to 9.7 and $13.5 \%$ of the stroke population, respectively ( $p=0.01$, Table 2). Mean age was similar (80.9 years in the SNDS vs. 82.4 years in the registry) although there was a greater, but nonsignificant, proportion of women in the registry ( 56 vs. $50 \%, p=0.34$ ).

In the registry, when we extended the population to all stroke patients with AF, 361 patients were identified (30.4\% of stroke patients) compared to 245 when considering cases 1 and 2 together for patients treated with OAC ( $20.3 \%$ of stroke patients).

\section{Discussion}

Overall, the proportion of stroke patients treated with OACs for AF was lower in the SNDS for the Dijon area compared to the Dijon Stroke Registry (13.4 [ $n=154]$ versus $20.3 \%$ [ $n=245], p<0.0001)$. This suggests that the identification of stroke cases treated with OACs for AF using the SNDS might be a useful approach but not sufficient.

Very few studies to date have compared administrative claims databases with a population-based registry, considered to be the gold standard, for cases of stroke patients with OAC-treated AF in the same geographical area [8]. Furthermore, it is difficult to compare our study with findings from other countries as the administrative claims databases may be constructed differently.

\section{Issues for the Identification of Stroke}

Before identifying patients treated with $\mathrm{OAC}$ for $\mathrm{AF}$ in the SNDS, we first needed to identify stroke cases. The validation of stroke cases identified in the SNDS has been partially achieved. Overall, the positive predictive value of stroke ICD-10 codes was found to be very high (around $90 \%)$ in a previous study [3]. However, this study was based on centers from 31 hospitals that participated on a voluntary basis. Another study was conducted in the area of Dijon and linked stroke patients registered in the PMSI after hospitalization to stroke cases identified in the Dijon Stroke Registry [9]. The sensitivity of stroke cases in the PMSI was estimated to be $77 \%$, with a predictive positive value of $69 \%$. As SNDS records all hospitalizations, stroke patients from the Dijon Stroke Registry who were hospitalized should be found in the PMSI databases. Differences between the registry and the PMSI were partially explained by the fact that potential stroke cases in the Dijon Stroke Registry are systematically reviewed and adjudicated by stroke-trained neurologist investigators. However, this previous study was based on data recorded from 
2004 to 2008. As coding policies have since changed, it is difficult to extrapolate these conclusions to our present results. Yet an improvement in sensitivity and predictive positive values was consistently observed over the study period, reaching 83 and $81 \%$, respectively, for the year 2008. The authors concluded that the PMSI databases were quite accurate and reliable to identify stroke cases.

In our study, the comparison of the distribution of stroke subtypes was restricted to ischemic, hemorrhagic, and undetermined strokes as the SNDS inaccurately records ischemic stroke subtypes. Moreover, the proportion of undetermined strokes was almost null in the Dijon Stroke Registry because all strokes that were initially classified as undetermined were subsequently reclassified by neurologists, which was not the case in the SNDS.

\section{Issues for the Identification of $A F$}

In the SNDS, we were able to study only AF treated with OAC as AF diagnoses are only available for hospital discharge data (i.e., inpatient diagnosis), not for outpatients. Indeed, the SNDS does not provide any clinical data such as the reasons for the complementary examinations or drug prescriptions, the results of these exams, or physicians' diagnoses in the outpatient context (i.e., not discharged from hospital). For this reason, we built an algorithm capable of predicting the indication for OAC treatment (AF vs. venous thromboembolism, including deep venous thrombosis and pulmonary embolism, as well as orthopedic or valvulopathy procedures) in an outpatient context, in accordance with data for the other covariates in the database $[5,10]$. In the outpatient context without $\mathrm{OAC}$, it is not feasible to discriminate AF patients from other cardiovascular diseases using healthcare data.

As OAC treatment indicated for AF is fully reimbursed in France whenever a patient goes to a pharmacy, this information is recorded in the SNDS. Furthermore, in our study, we used the date of drug delivery. Accordingly, while we cannot be sure that patients did indeed take the prescribed OAC, we can be sure that it was delivered to them. In the Dijon Stroke Registry, AF was reliably identified as we had access to the complete medical file of patients. However, the registry only recorded the prescription of OAC treatment at discharge, and we had no information as to whether the patients effectively received their treatment. This difference might explain the discrepancies in our results, especially the difference in the mean age for case 1 patients between the SNDS and the registry. Indeed, we observed that case 1 patients in the registry were older than those identified in the SNDS, which could be explained by the fact that only younger patients actually collect their OAC treatment in a pharmacy. Adherence to OAC treatment could not be accurately recorded in the SNDS or the registry, while information on OAC dosage was not available.

Finally, AF history was limited to 3 years in the SNDS, whereas the Dijon Stroke Registry recorded AF diagnosis for the patient's lifetime. This might explain the lower rates of case 2 patients in the SNDS compared to the registry.

\section{Major Advantage of the Dijon Stroke Registry}

Although the SNDS may be a useful database to study stroke patients with OAC-treated AF, the Dijon Stroke Registry recorded all AF stroke patients regardless of OAC treatment. These patients accounted for $30.4 \%$ of stroke patients for the years 2013-2017, whereas stroke cases with OAC-treated AF represented only $20.3 \%$ of stroke patients in the registry. Furthermore, AF was recorded as a comorbidity in the SNDS, whereas the type of investigation used in the registry allowed us to accurately assess whether AF was the cause of stroke. This underlines the importance of maintaining and developing population-based stroke registries for the surveillance of AFrelated strokes.

Of the $30.4 \%$ stroke patients with AF, half were prescribed OAC at discharge in the Dijon Stroke Registry (data not shown). Several reasons might explain this rate of anticoagulation. First, 15\% of AF strokes were hemorrhagic strokes and could be prescribed any anticoagulation. Second, we took into account only oral anticoagulation prescription. When extended to all anticoagulants, including heparin therapy, we observed that almost $60 \%$ of AF stroke received anticoagulation therapy at discharge. The remaining $25 \%$ of AF stroke not receiving anticoagulant prescription at discharge might have contraindication, or initiation or resumption of OAC may have been delayed for several days to avoid the risk of hemorrhagic transformation according to clinical guidelines [11].

\section{Limitations}

Some limitations of this study must be acknowledged. First, although the algorithm used to identify AF patients newly treated with OAC in the SNDS had an internal validation, it has not received an external validation yet. The algorithm might be improved in the future, allowing higher rates of AF identification in hospitalized stroke patients. Second, information about OAC treatment before stroke was not based on specific dosage neither in the SNDS nor the Dijon Stroke Registry. Therefore, we cannot be sure that patients were adherent to their treatment. 
Finally, residents of Dijon all live in an urban area. Observations from the Dijon Stroke Registry could therefore not be fully extrapolated to the whole French population, particularly rural areas.

\section{Conclusion}

Administrative health databases can be a useful tool to identify hospitalized stroke patients with OAC-treated AF. The results observed in this study need proper validation to link the Dijon Stroke Registry with the SNDS databases. However, the registry remains the gold standard to fully study stroke cases with AF.

\section{Statement of Ethicss}

In line with French governmental regulations and the National Ethics Committee, no patient consent was required for the SNDS databases. Database used contained de-identified patient information.
The Dijon Stroke Registry was approved by following national ethics boards: the Comité d'Evaluation des Registres (French National Committee of Registers), Santé Publique France (French Institute for Public Health Surveillance), and the Commission Nationale Informatique et Liberté (French Data Protection Authority). In accordance with the French legislation, boards waived the need for written patient consent.

\section{Conflict of Interest Statement}

Yannick Béjot reports personal fees from AstraZeneca, BMS, Pfizer, Medtronic, MSD France, Amgen, Servier, and BoehringerIngelheim.

\section{Funding Sources}

Santé Publique France (French Institute for Public Health Surveillance), INSERM, and University Hospital of Dijon.

\section{References}

1 Kirchhof P, Benussi S, Kotecha D, Ahlsson A, Atar D, Casadei B, et al. 2016 ESC Guidelines for the management of atrial fibrillation developed in collaboration with EACTS. Europace. 2016;18(11):1609-78.

2 Tuppin P, Rudant J, Constantinou P, Gastaldi-Ménager C, Rachas A, de Roquefeuil L, et al. Value of a national administrative database to guide public decisions: from the système national d'information interrégimes de l'Assurance Maladie (SNIIRAM) to the système national des données de santé (SNDS) in France. Rev Epidemiol Sante Publique. 2017; 65(Suppl 4):S149-67.

3 Giroud M, Hommel M, Benzenine E, Fauconnier J, Béjot Y, Quantin C. Positive predictive value of french hospitalization discharge codes for stroke and transient ischemic attack. Eur Neurol. 2015;74(1-2):92-9.

4 Lecoffre C, de Peretti C, Gabet A, Grimaud O, Woimant F, Giroud M, et al. National trends in patients hospitalized for stroke and stroke mortality in France, 2008 to 2014. Stroke. 2017 Nov;48(11):2939-45.
5 Gabet A, Chatignoux E, Billionnet C, Maura G, Giroud M, Béjot Y, et al. Annual rate of newly treated atrial fibrillation by age and gender in France, 2010-2016. Eur J Epidemiol. 2019.

6 Feigin V, Norrving B, Sudlow CLM, Sacco RL. Updated criteria for population-based stroke and transient ischemic attack incidence studies for the 21st century. Stroke. 2018;49(9): 2248-55.

7 WHO. The world health report 2000: health systems improving performance. Geneva: WHO.

8 Sung SF, Hsieh CY, Lin HJ, Chen YW, Yang YH, Li CY. Validation of algorithms to identify stroke risk factors in patients with acute ischemic stroke, transient ischemic attack, or intracerebral hemorrhage in an administrative claims database. Int J Cardiol. 2016;215: $277-82$.
9 Aboa-Eboule C, Mengue D, Benzenine E, Hommel M, Giroud M, Béjot Y, et al. How accurate is the reporting of stroke in hospital discharge data? A pilot validation study using a population-based stroke registry as control. J Neurol. 2013;260(2):605-13.

10 Billionnet C, Alla F, Bérigaud É, Pariente A Maura G. Identifying atrial fibrillation in outpatients initiating oral anticoagulants based on medico-administrative data: results from the French national healthcare databases. Pharmacoepidemiol Drug Saf. 2017;26(5): 535-43.

11 Klijn CJ, Paciaroni M, Berge E, Korompoki E, Kõrv J, Lal A, et al. Antithrombotic treatment for secondary prevention of stroke and other thromboembolic events in patients with stroke or transient ischemic attack and nonvalvular atrial fibrillation: a European Stroke Organisation guideline. Eur Stroke J. 2019; 4(3):198-223. 